Pacific Journal of Mathematics

SOME CHARACTERIZATIONS OF EXPONENTIAL-TYP 


\title{
SOME CHARACTERIZATIONS OF EXPONENTIAL-TYPE DISTRIBUTIONS
}

\author{
E. M. Bolger ${ }^{1}$ and W. L. HARKNESS
}

Let $f=\{f(x ; \delta)=\exp [x \delta+q(\delta)], \delta \in(a, b)\}$ be a family of exponential-type probability density-functions (exp. p.d.f.'s) with respect to a $\sigma$-finite measure $\mu$. Let $M(t ; \delta), a-\delta<t<$ $b-\delta$, denote the moment generating function (m.g.f.) corresponding to $f(x ; \delta) \in f$, and let $c(t ; \delta)=\ln M(t ; \delta)=$ $\sum_{k=1}^{\infty} \lambda_{k}(\delta) t^{k} / k$ ! be the cumulative generating function. The main results pertain to characterizations of certain exp. p.d.f.'s in terms of the cumulants $\lambda_{k}(\delta)$. First, it is shown that if $M\left(t ; \delta_{0}\right)$ is the m.g.f., respectively, of a degenerate, Poisson, or normal law for some $\delta_{0} \in(a, b)$, then $M(t ; \delta)$ is the m.g.f. of the given law for all $\delta \in(a, b)$, and that infinite divisibility (inf. div) of $M\left(t ; \delta_{0}\right)$ for some $\delta_{0}$ implies inf. div. for all $\delta$. Further, it is shown that if $\varphi(t)$ is a nondegenerate, inf. div. characteristic function (ch. f.) with finite fourth cumulant $\lambda_{4}$, then $\lambda_{4}=0$ if and only if $\varphi(t)$ is the ch.f. of a normal law, while if $\lambda_{4}=a \lambda_{3}=a^{2} \lambda_{2} \neq 0$, then $\varphi(t)$ is the ch.f. of a Poisson law. Combining these results, it follows that if $M\left(t ; \delta_{0}\right)$ is inf. div., and nondegenerate, with $\lambda_{4}\left(\delta_{0}\right)=0$, then $M(t ; \delta)$ is the m.g.f. of a normal law for all $\delta \in(a, b)$. A similar result characterizes the Poisson law. Finally, it is proved that the normal law is the unique exp. p.d.f. which is symmetric.

An exponential-type family of distributions is defined by probability densities of the form

$$
f(y ; \delta)=\exp [y \delta+q(\delta)], \quad a<\delta<b
$$

with respect to a $\sigma$-finite measure $\mu$ over a Euclidean sample space $(\mathfrak{X}, \mathfrak{A})$. It is known $([1]$, p. 51$)$ that the set of parameter points $\delta$ such that $\int \exp [\delta y] d \mu(y)<\infty$, is an interval (finite or not). The binomial, Poisson, normal, gamma, and negative binomial disiributions provide familiar examples of exponential-type distributions.

A few structural properties for this family are considered. Section 2 contains some useful lemmas which are applied in $\S 3$ to obtain some characterizations of the Poisson and normal distributions.

2. Some lemmas, Patil [3] has shown that a collection of d.f.'s $\{F(x ; \delta): \delta \in(a, b)\}$ is of exponential-type if and only if the

Received March 12, 1964 and in revised form July 27, 1964.

1 Now at Bucknell University. 
cumulants, $\lambda_{k}(\delta)$, exist for all $k$ and satisfy

$$
\lambda_{k}(\delta)=\frac{d \lambda_{k-1}(\delta)}{d \delta} \quad \text { for } k=2,3,4, \cdots
$$

Further, he has shown [3, equation (12)] that $M(t ; \delta)$ is the moment generating function of an exponential d.f. if and only if $M(t ; \delta)=$ $\exp \{q(\delta)-q(\delta+t)\}$. Lehmann ([1], p. 52) has shown that $e^{-q(\delta)}$ is an analytic function of $\delta$ for $a<R e \delta<b$. It follows that $q(\delta)$ is. analytic for $a<\operatorname{Re} \delta<b$. Then $\lambda_{k}(\delta)$ is analytic for $a<\operatorname{Re} \delta<b$. and $k \geqq 1$. Hence, if $\delta_{0} \in(a, b)$, there is a neighborhood $\Delta$ of $\delta_{0}$ such that

$$
\lambda_{j}(\delta)=\sum_{k=0}^{\infty} \frac{\lambda_{j+k}\left(\delta_{0}\right)\left(\delta-\delta_{0}\right)^{k}}{k !} \quad \text { for } \delta \in \Delta
$$

Lemma 1. If $M\left(t ; \delta_{0}\right)$ is degenerate for some $\delta_{0} \in(a, b)$, then $M(t ; \delta)$, is degenerate for all $\delta \in(a, b)$.

Proof. $M\left(t ; \delta_{0}\right)$ degenerate implies $\lambda_{j}\left(\delta_{0}\right)=0$ for $j \geqq 2$. Write

$$
\lambda_{2}(\delta)=\sum_{j=0}^{\infty} \frac{\lambda_{2+j}\left(\delta_{0}\right)\left(\delta-\delta_{0}\right)^{j}}{j !} \quad \text { for } \delta \in \Delta .
$$

Thus, $\lambda_{2}(\delta) \equiv 0$ for $\delta \in \Delta$. Since $\lambda_{2}(\delta)$ is analytic for $a<R e \delta<b$, we have $\lambda_{2}(\delta) \equiv 0$ for $\delta \in(a, b)$ and the conclusion follows.

CoROLlary. If $\lambda_{2}\left(\delta_{0}\right)$ is different from zero for at least one $\delta_{0} \in(a, b)$, then $\lambda_{2}(\delta)$ is different from zero for all $\delta \in(a, b)$.

Lemma 2. If $M\left(t ; \delta_{0}\right)$ is the m.g.f. of a Poisson type distribution for some $\delta_{0} \in(a, b)$, then $M(t ; \delta)$ is the m.g.f. of a Poisson type distribution for all $\delta \in(a, b)$.

Proof. By assumption.

$$
M\left(t ; \delta_{0}\right)=\exp \left\{\frac{\lambda_{2}\left(\delta_{0}\right)}{c^{2}}\left(e^{c t}-1\right)+\left(\lambda_{1}\left(\delta_{0}\right)-\frac{\lambda_{2}\left(\delta_{0}\right)}{c}\right) t\right\}
$$

and

$$
\lambda_{j}\left(\delta_{0}\right)=c^{j-2} \lambda_{2}\left(\delta_{0}\right) \quad \text { for } j \geqq 2 .
$$

If it can be shown that

$$
\lambda_{j}(\delta)=c^{j-2} \lambda_{2}(\delta) \quad \text { for } j \geqq 2
$$

and all $\delta \in(a, b)$, then the Lemma will follow. The proof of (3) is by 
induction on $j$. Let $h(\delta)=\lambda_{3}(\delta)-c \lambda_{2}(\delta)$. Now $h(\delta)$ is analytic for $a<\operatorname{Re} \delta<b$. Furthermore, $h\left(\delta_{0}\right)=0$, and

$$
\begin{aligned}
h^{(k)}\left(\delta_{0}\right) & =\lambda_{3+k}\left(\delta_{0}\right)-c \lambda_{2+k}\left(\delta_{0}\right) \\
& =c^{k+1} \lambda_{2}\left(\delta_{0}\right)-c c^{k} \lambda_{2}\left(\delta_{0}\right) \\
& =0 .
\end{aligned}
$$

It follows that $h(\delta) \equiv 0$ for $\delta \in(a, b)$. So $\lambda_{3}(\delta)=c \lambda_{2}(\delta)$. Now, assume $\lambda_{j}(\delta)=c^{j-2} \lambda_{2}(\delta)$. Differentiation of both sides yields

$$
\lambda_{j+1}(\delta)=c^{j-2} \lambda_{3}(\delta)=c^{j-2} c \lambda_{2}(\delta)=c^{(j+1)-2} \lambda_{2}(\delta) .
$$

This completes the proof of (3). It follows that

$$
M(t ; \delta)=\exp \left\{\frac{\lambda_{2}(\delta)}{c^{2}}\left(e^{c t}-1\right)+\left(\lambda_{1}(\delta)-\frac{\lambda_{2}(\delta)}{c}\right) t\right\} .
$$

LEMMA 3. If $M\left(t ; \delta_{0}\right)$ is normal for some $\delta_{0} \in(a, b)$, then $M(t ; \delta)$ is normal for all $\delta \in(a, b)$.

Proof. Since $M\left(t ; \delta_{0}\right)$ is normal, $\lambda_{2}\left(\delta_{0}\right) \neq 0$ and $\lambda_{j}\left(\delta_{0}\right)=0$ for $j \geqq 3$. Write for $\delta \in \Delta$,

$$
\lambda_{3}(\delta)=\sum_{j=0}^{\infty} \frac{\lambda_{3+j}\left(\delta_{0}\right)\left(\delta-\delta_{0}\right)^{j}}{j !}=0
$$

Then $\lambda_{3}(\delta) \equiv 0$ for $\delta \in(a, b)$. Because of (2) it follows that $\lambda_{j}(\delta)=0$ for $j \geqq 3$. Finally, $\lambda_{2}\left(\delta_{0}\right) \neq 0$ implies $\lambda_{2}(\delta) \neq 0$ for any $\delta \in(a, b)$.

LeMmA 4. If $M\left(t ; \delta_{0}\right)$ is infinitely divisible for some $\delta_{0} \in(a, b)$, then $M(t ; \delta)$ is infinitely divisible for all $\delta \in(a, b)$.

Proof. If $\lambda_{2}\left(\delta_{0}\right)=0$, the result follows from Lemma 1. So assume $\lambda_{2}(\delta) \neq 0$ for any $\delta \in(a, b)$. Now, (Lukacs [2]), there exists a distribution $G\left(x ; \delta_{0}\right)$ such that

$$
\lambda_{2}\left(\delta_{0}+t\right) / \lambda_{2}\left(\delta_{0}\right)=\int e^{x t} d G\left(x ; \delta_{0}\right)
$$

for $t \in\left(a-\delta_{0}, b-\delta_{0}\right)$. Let $\delta_{1}$ be an arbitrary element of $(a, b)$. If $t \in\left(a-\delta_{1}, b-\delta_{1}\right)$, then $t+\delta_{1} \in(a, b)$ and $t+\delta_{1}-\delta_{0} \in\left(a-\delta_{0}, b-\delta_{0}\right)$. Hence, for $t \in\left(a-\delta_{1}, b-\delta_{1}\right)$

$$
\begin{aligned}
\frac{\lambda_{2}\left(\delta_{1}+t\right)}{\lambda_{2}\left(\delta_{1}\right)} & =\frac{\lambda_{2}\left[\delta_{0}+\left(t_{1}+\delta_{1}-\delta_{0}\right)\right]}{\lambda_{2}\left(\delta_{1}\right)} \\
& =\frac{\lambda_{2}\left(\delta_{0}\right)}{\lambda_{2}\left(\delta_{1}\right)} \int e^{\left(t+\delta_{1}-\varepsilon_{0}\right)} d G\left(x ; \delta_{0}\right)=\int e^{t x} d G_{1}\left(x ; \delta_{0}\right)
\end{aligned}
$$


where $d G_{1}\left(x ; \delta_{0}\right)=\left(\lambda_{2}\left(\delta_{0}\right) / \lambda_{2}\left(\delta_{1}\right)\right) e^{\left(\delta_{1}-\delta_{0}\right) x} d G\left(x ; \delta_{0}\right)$. It is easy to see that $G_{1}\left(x ; \delta_{0}\right)$ is a distribution function. Thus,

$$
\lambda_{2}\left(\delta_{1}+t\right) / \lambda_{2}\left(\delta_{1}\right)
$$

is a moment generating function for $t \in\left(a-\delta_{1}, b-\delta_{1}\right)$. Hence, $M\left(t ; \delta_{1}\right)$ is infinitely divisible. Since $\delta_{1}$ is an arbitrary element of $(a, b), M(t ; \delta)$ is infinitely divisible for all $\delta \in(a, b)$.

In the following two lemmas, we assume that $f(t)$ is a nondegenerate, infinitely divisible characteristic function (ch. f.) and $\varphi(t)=\log f(t)$ has four derivatives at $t=0$. Let

$$
\lambda_{j}=\frac{i^{j} d^{j} \varphi(0)}{d t^{j}}, \quad j=1,2,3,4 .
$$

From the results of Shapiro [4], it is easily deduced that $-\left(1 / \lambda_{2}\right)\left(d^{2} \varphi(t) / d t^{2}\right)$ is the characteristic function of a d.f. with mean $\lambda_{3} / \lambda_{2}$ and variance $\left(\lambda_{2} \lambda_{4}-\lambda_{3}^{2}\right) / \lambda_{2}^{2}$.

LEMMA 5. If $\lambda_{4}=0$, then $f(t)$ is the characteristic function of a normal distribution.

Proof. $-\left(1 / \lambda_{2}\right)\left(d^{2} \varphi(t) / d t^{2}\right)$ is a characteristic function of a distribution with mean $\lambda_{3} / \lambda_{2}$ and variance $\left(\lambda_{2} \lambda_{4}-\lambda_{3}^{2}\right) / \lambda_{2}^{2}$. Thus $\lambda_{4}=0$ implies $\lambda_{3}=0$ since the variance is nonnegative. Therefore, $-\left(1 / \lambda_{2}\right)\left(d^{2} \varphi(t) / d t^{2}\right)$ is the ch. $f$. of a degenerate distribution with mean 0 . Hence,

$$
\frac{-1}{\lambda_{2}} \frac{d^{2} \varphi(t)}{d t^{2}} \equiv 1
$$

and, it follows that $\varphi(t)=i \lambda_{1} t-\left(\lambda_{2} t^{2} / 2\right)$ for all $t$.

Note that the single assumption that $\lambda_{4}=0$ does not suffice to ensure normality since the binomial distribution, while not infinitely divisible, with $p q=1 / 6$ has $\lambda_{4}=0$.

LEMmA 6. If $\lambda_{4}=a \lambda_{3}=a^{2} \lambda_{2} \neq 0$, and $f(t)$ is infinitely divisible, then $f(t)$ is the characteristic function of a Poisson type distribution.

Proof. $-\left(1 / \lambda_{2}\right)\left(d^{2} \varphi(t) / d t^{2}\right)$ is the ch.f. of a distribution with mean $\lambda_{3} / \lambda_{2}=a$ and variance $\left(\lambda_{2} \lambda_{4}-\lambda_{3}^{2}\right) / \lambda_{2}^{2}=\left(a^{2} \lambda_{2}^{2}-a^{2} \lambda_{2}^{2}\right) / \lambda_{2}^{2}=0$. So, $-\left(1 / \lambda_{2}\right)\left(d^{2} \varphi(t) / d t^{2}\right)$ is a ch.f. of a degenerate distribution with mean a. That is,

$$
-\frac{1}{\lambda_{2}} \frac{d^{2} \varphi(t)}{d t^{2}}=e^{i a t}
$$

It follows that 


$$
\varphi(t)=\frac{\lambda_{2}}{a^{2}}\left(e^{i a t}-1\right)+i\left(\lambda_{1}-\frac{\lambda_{1}}{a}\right) t .
$$

REMARK 1. It is not sufficient to assume infinite divisibility and $\lambda_{3}=\lambda_{4} \neq 0$.

ExAMPLE. Let $\varphi(t)=\lambda\left(e^{i t}-1\right)+i \lambda t-\left(t^{2} / 2\right)$. Then $\lambda_{3}=\lambda_{4}=\lambda \neq 0$. $\varphi(t)$ is the ch.f. of the composition of normal and Poisson distributions.

REMARK 2. It is not sufficient to assume infinite divisibility and $\lambda_{2}=\lambda_{3} \neq 0$.

ExAMPLE. Let $\varphi(t)=e^{2 i t}-1-2 t^{2}$. Then $\lambda_{2}=\lambda_{3}=8$.

REMARK 3. It is not sufficient to assume $\lambda_{2}=\lambda_{3}=\lambda_{4} \neq 0$.

EXAMPLE. Let $x_{0}=(1+\sqrt{13}) / 2$ and $x_{1}=1-x_{0}$. Let $p_{0}=$ $\left(x_{0}-1\right) /\left(2 x_{0}-1\right)$ and $p_{1}=1-p_{0}$. It is easy to see that $0<p_{0}, p_{1}<1$. Let $g_{1}(t)=e^{i x_{0} t} p_{0}+e^{i x_{1} t} p_{1}$ and $g_{2}(t) \equiv 1$. Then, if

$$
g(t)=\frac{1}{3} g_{1}(t)+\frac{2}{3} g_{2}(t),
$$

it follows by direct computation that $\lambda_{2}=\lambda_{3}=\lambda_{4}=1$. Here, $g(t)$ is obviously not an infinitely divisible ch.f. .

\section{Characterization of the normal aud Poisson distributions.}

THEOREM 1. If $M\left(t ; \delta_{0}\right)$ is infinitely divisible and nondegenerate, and if $\lambda_{4}\left(\delta_{0}\right)=0$, then $M(t ; \delta)$ is the m.g.f. of a normal distribution, for all $\delta \in(a, b)$.

Proof. By Lemma 5, $M\left(t ; \delta_{0}\right)$ is the m.g.f. of a normal distribution. Then by Lemma 3, the conclusion holds for all $\delta \in(a, b)$.

The family of normal distributions has the property that all its members are symmetric distributions. This means that all central moments of odd order vanish; in particular, the third central moment $\mu_{3}=\lambda_{3}$, must vanish. The next theorem, which follows easily from equation (2) and Lemma 3, implies that the normal law is the unique exponential-type distribution which is symmetric.

THEOREM 2. Let $f=\left\{F(x ; \delta)=\int_{-\infty}^{x} e^{\nu \delta+q(\delta)} d \mu(y) ; \delta \in(a, b)\right\}$ be $a$ family of exponential-type distributions, and assume that $\lambda_{3}(\delta)=0$ 
for all $\delta \in(a, b)$ and $\lambda_{2}\left(\delta_{0}\right)>0$ for some $\delta_{0} \in(a, b)$. Then $f$ is $a$ family of normal distributions.

The following question now arises: If, for some $\delta_{0} \in(a, b), M\left(t ; \delta_{0}\right)$ is infinitely divisible and $\lambda_{3}\left(\delta_{0}\right)=0$, must $M(t ; \delta)$ be normal? The answer is no.

ExAmPLe. Let $N(t)=e^{-t+t^{2} / 2}$ for $-\infty<t<\infty$,

$$
P(t)=\int_{0}^{t} \int_{0}^{s} N(y) d y d s,
$$

and $N_{1}(t)=e^{P(t)}$. Then, (Lukacs [2]), $N_{1}(t)$ is an infinitely divisible moment generating function. Clearly,

$$
M(t ; \mu)=\frac{N_{1}(t+\mu)}{N_{1}(\mu)}=e^{-\log N_{1}(\mu)+\log N_{1}(\mu+t)}
$$

is an exponential-type moment generating function. It is easy to see that $M(t ; \mu)$ is infinitely divisible. Now

$$
\begin{aligned}
\lambda_{3}(\mu) & =\left.\frac{d^{3} \log M(t ; \mu)}{d t^{3}}\right|_{t=0} \\
& =\left.\frac{d^{3} P(t+\mu)}{d t^{3}}\right|_{t=0}=\left.\frac{d N(t+\mu)}{d t}\right|_{t=0}=\frac{d N(\mu)}{d \mu} \\
& =(-1+\mu) e^{-\mu+\mu^{2} / 2}
\end{aligned}
$$

so that $\lambda_{3}(1)=0$. However, $\lambda_{3}(\mu)$ is not identically zero so that $M(t ; \mu)$ is not the m.g.f. of a normal distribution for any value of $\mu$. [For $M\left(t ; \mu_{0}\right)$ normal would imply $M(t ; \mu)$ normal for all $\mu$ which, in turn, would imply $\lambda_{3}(\mu) \equiv 0$.]

THEOREM 3. If $M\left(t ; \delta_{0}\right)$ is infinitely divisible for some $\delta_{0} \in(a, b)$, and if $\lambda_{4}\left(\delta_{0}\right)=c \lambda_{3}\left(\delta_{0}\right)=c^{2} \lambda_{2}\left(\delta_{0}\right) \neq 0$, then $M(t ; \delta)$ is the m.g.f. of $a$ Poisson type distribution for all $\delta \in(a, b)$.

Proof. This follows directly from Lemmas 2 and 6.

THEOREM 4. If $\lambda_{3}(\delta) \equiv c \lambda_{2}(\delta)$ for all $\delta \in(a, b)$ where $\lambda_{2}(\delta)$ and $\lambda_{3}(\delta)$ are cumulants of an exponential-type distribution, then $M(t ; \delta)$ is the m.g.f. of a Poisson type distribution.

Proof. First we show by induction that

$$
\lambda_{j+2}(\delta)=c^{j} \lambda_{2}(\delta) \text {. }
$$

By assumption, this is true for $j=1$. Assume now that $\lambda_{j+2}(\delta)=$ 
$c^{j} \lambda_{2}(\delta)$. Differentiating both sides, we get

$$
\lambda_{j+3}(\delta)=c^{j} \lambda_{3}(\delta)=c^{j+1} \lambda_{2}(\delta) \text {. }
$$

Then,

$$
\log M(t ; \delta)=\frac{\lambda_{2}(\delta)}{c^{2}}\left(e^{c t}-1\right)+\left(\lambda_{1}(\delta)-\frac{\lambda_{2}(\delta)}{c}\right) t
$$

REMARK. Let $\delta_{0}, \delta_{1} \in(a, b)$. Many of the preceding results would be trivial if there existed constants $c, d$ with $c \neq 0$ such that

$$
M\left(t ; \delta_{0}\right)=e^{d t} M\left(c t, \delta_{1}\right) \text {. }
$$

However, that this is not always the case is shown by taking

$$
M(t ; \delta)=e^{e^{\delta}\left(e^{t}-1\right)}, \quad t, \delta \in(-\infty, \infty) .
$$

\section{REFERENCES}

1. E. L. Lehmann, Testing Statistical Hypotheses, John Wiley, New York, 1959.

2. Eugene Lukacs, Characteristic functions, Hafner, New York, 1960.

3. G. P. Patil, A characterization of the exponential-type distribution, Biometrika 50 (1963), 205-207.

4. J. M. Shapiro, A condition for existence of moments of infinitely divisible distributions, Canad. J. Math. 8 (1956), 69-71.

The Pennsylvania State University 



\section{PACIFIC JOURNAL OF MATHEMATICS}

EDITORS

H. SAMELSON

Stanford University

Stanford, California

R. M. BLUMENTHAL

University of Washington

Seattle, Washington 98105
*J. DUGUNDJI

University of Southern California

Los Angeles, California 90007

RICHARD ARENS

University of California

Los Angeles, California 90024

\section{ASSOCIATE EDITORS}

E. F. BECKENBACH

B. H. NeumanN

F. WOLF

K. YosidA

\section{SUPPORTING INSTITUTIONS}

UNIVERSITY OF BRITISH COLUMBIA

CALIFORNIA INSTITUTE OF TECHNOLOGY

UNIVERSITY OF CALIFORNIA

MONTANA STATE UNIVERSITY

UNIVERSITY OF NEVADA

NEW MEXICO STATE UNIVERSITY

OREGON STATE UNIVERSITY

UNIVERSITY OF OREGON

OSAKA UNIVERSITY

UNIVERSITY OF SOUTHERN CALIFORNIA
STANFORD UNIVERSITY

UNIVERSITY OF TOKYO

UNIVERSITY OF UTAH

WASHINGTON STATE UNIVERSITY

UNIVERSITY OF WASHINGTON

AMERICAN MATHEMATICAL SOCIETY CHEVRON RESEARCH CORPORATION

TRW SYSTEMS

NAVAL ORDNANCE TEST STATION 


\section{Pacific Journal of Mathematics}

\section{Vol. 16, No. $1 \quad$ November, 1966}

Larry Armijo, Minimization of functions having Lipschitz continuous first

partial derivatives ............................... 1

Edward Martin Bolger and William Leonard Harkness, Some

characterizations of exponential-type distributions.............. 5

James Russell Brown, Approximation theorems for Markov operators ...... 13

Doyle Otis Cutler, Quasi-isomorphism for infinite Abelian p-groups ...... 25

Charles M. Glennie, Some identities valid in special Jordan algebras but not valid in all Jordan algebras .......................... 47

Thomas William Hungerford, A description of $\operatorname{Mult}_{i}\left(A^{1}, \cdots, A^{n}\right)$ by

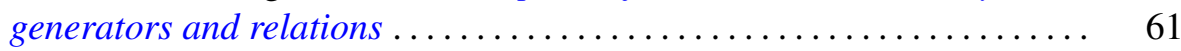

James Henry Jordan, The distribution of cubic and quintic non-residues ....

Junius Colby Kegley, Convexity with respect to Euler-Lagrange differential operators................................... 87

Tilla Weinstein, On the determination of conformal imbedding ......... 113

Paul Jacob Koosis, On the spectral analysis of bounded functions ........ 121

Jean-Pierre Kahane, On the construction of certain bounded continuous functions ................................... 129

V. V. Menon, A theorem on partitions of mass-distribution ........... 133

Ronald C. Mullin, The enumeration of Hamiltonian polygons in triangular

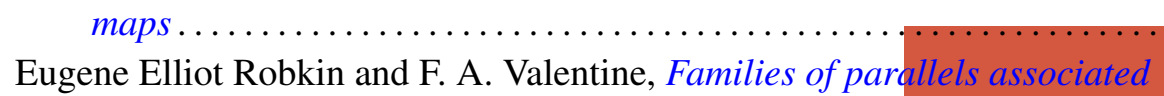

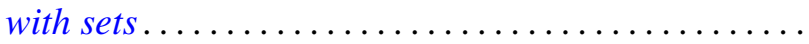

Melvin Rosenfeld, Commutative F-algebras

A. Seidenberg, Derivations and integral closure

S. Verblunsky, On the stability of the set of exponents of a Cauchy

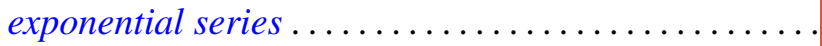

Herbert Walum, Some averages of character sums 